\title{
THE PARTON-LIKE MODEL AND PHOTON ENERGY SPECTRA IN $B$ DECAYS
}

\author{
Ilya NARODETSKII \\ Institute of Theoretical and Experimental Physics
}

117218 Moscow, RF

\begin{abstract}
We discuss a new parton-like formula, which establishes a simple connection between the electroweak decay rate $\Gamma\left(\bar{B} \rightarrow X_{s} \gamma\right)$ and the rate of a free $b$-quark decay. Observable spectra are described through folding quark spectra with a $b$ quark light-front (LF) distribution function. Using various distribution functions derived from current constituent quark models, and neglecting perturbative corrections we compute the photon energy spectra and the moments of the shape function. It is shown that the parton-like approach is fully consistent with the Heavy Quark Effective Theory (HQET) provided the $b$-quark constituent mass is redefined in the way similar to that used in HQET to define the pole mass of the $b$ quark. In this way the correction to first order in $1 / m_{b}$ can be eliminated from the total width in agreement with the general statement of HQET. We have also found that the photon energy spectra calculated in the LF approach agree well with the ones obtained in the ACM model, provided the same distribution function is used as input in both cases. In spite of the simplicity of the model our results show a fair good agreement both with the HQET predictions and available experimental data.
\end{abstract}

\section{INTRODUCTION}

Studying of the photon spectrum in the weak radiative $\bar{B} \rightarrow X_{s} \gamma$ is important for understanding how precisely the total rate can be predicted in the presence of an experimental cut on the photon energy E. Experimentally, because of background from nonleptonic charged current processes $b \rightarrow c \bar{u} d+\gamma$ or $b \rightarrow u \bar{u} d+\gamma$, which have a typical Bremsstrahlung spectrum, only the high energy part of the photon spectrum can be detected with the present experimental cut $E>2.1 \mathrm{GeV}$ at CLEO [i1].1. Moments of the photon energy spectrum may be used to measure the HQET parameters which determine the

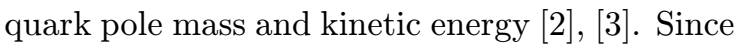
the $b$-quark is heavy compared to the QCD scale, the inclusive $B \rightarrow X_{s} \gamma$ decay rate can be calcu-

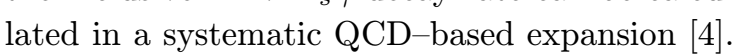

However, near the end point an important nonperurbative effect due to the soft interactions of the $b$-quark with the light constituents has to be included. This so-called "Fermi motion" can be included in the heavy quark expansion by resumming an infinite set of leading-twist contributions into a shape function $F(x)$ [inj, where a scaling variable is defined as $x=\left(2 E-m_{b}\right) / \bar{\Lambda}$ with $\bar{\Lambda}=M_{B}-m_{b}$. A model independent determination of the shape function is not available at the present time, however it may be possible to address this issue using lattice QCD [ín]. Ansätz for the shape function constrained by the information on its few first moments has been recently

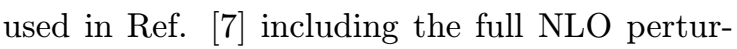
bative QCD corrections.

As to phenomenological analyses of the photon spectra up to now they have been solely based on the ACM model [8], [9] 
proach to consideration of the inclusive semileptonic $b \rightarrow c, u$ transitions was suggested in Refs. [1] $10 \overline{0}]-[1 \overline{1} 2 \overline{1}]$ and has been recently refined in [1] This approach treats the heavy hadron as a bound state of the heavy quark and a spectator, with a certain momentum distribution. Observable spectra are described through folding quark spectra with a LF distribution function $\left|\psi\left(\xi, p_{\perp}^{2}\right)\right|^{2}$, which represents the probability to find the $b$ quark carrying a LF fraction $\xi$ and a transverse relative momentum squared $p_{\perp}^{2}=\left|\mathbf{p}_{\perp}\right|^{2}$.

Some of the dynamical features of this model get obscured by the integration over the lepton energy. They are seen in a cleaner way in the spectrum of the photons in the radiative $\bar{B} \rightarrow$ $X_{s} \gamma$ transitions. In this talk, we extend the work of Ref. [1] 1 in to compute the non-perturbative corrections to the photon spectrum and the $\bar{B} \rightarrow$ $X_{s} \gamma$ inclusive rate ${ }^{1}$. We strive to implement the $B$-meson wave function effects on the photon energy and the invariant mass distributions of the hadrons recoiling against the photon. We will also study the comparison between the photon spectra $d \Gamma / d E$ calculated in the LF and ACM approaches and will show that the discrepancy between the two is very small numericallly.

\section{Inclusive Photon Spectrum}

Similar to the ACM model the LF quark model treats the beauty meson $B$ as consisting of the heavy $b$ quark plus a spectator quark. Both quarks have fixed masses, $m_{b}$ and $m_{s p}$, though. This is at variance with the ACM model, that has been introduced in order to avoid the notion of the heavy quark mass at all.

The $b \rightarrow s \gamma$ transition at the fundamental level is generated by electroweak penguins [1 1150$]$, [1]6]. In the leading logarithmic approximation the decay $b \rightarrow s \gamma$ is described by the effective Lagrangian

$$
\mathcal{L}_{\text {eff }}=\frac{4 G_{F}}{\sqrt{2}} V_{t b} V_{t s}^{*} c_{7}(\mu) \bar{m}_{b}(\mu) O_{7},
$$

where $O_{7}=\left(e / 32 \pi^{2}\right) \bar{u}_{s} \sigma_{\mu \nu}\left(1+\gamma_{5}\right) u_{b} F^{\mu \nu}$. In equation $\left(2 . i^{1}\right) \bar{m}_{b}(\mu)$ and $c_{7}(\mu)$ are running $b$

\footnotetext{
${ }^{1} \mathrm{~A}$ preview of this work can be found in [1] 10.
}

quark mass and the Wilson coefficient, respectively, evaluated at a subtraction point $\mu$. The strange quark mass will be neglected throughout this paper; it only enters the final results quadratically as $m_{s}^{2} / m_{b}^{2}$. For simplicity we neglect contributions of other operators in the effective Lagrangian which appear at next-to-leading order.

To calculate the decay rate $\bar{B} \rightarrow X_{s} \gamma$ we use the approach of Ref. [1] which is based on the hypothesis of quark-hadron duality. This hypothesis assumes that the sum over all possible strange final states $X_{s}$ can be modeled by the decay width of an on-shell $b$ quark into on-shell $c$-quark weighted with the $b$-quark distribution function $f\left(\xi, p_{\perp}^{2}\right)$. Going through the intermediate steps (for the detail see Ref. [1] $\left.{ }^{1}\right]$ ) we obtain for the partial decay rate of the inclusive decay $B \rightarrow X_{s} \gamma$

$$
d \Gamma=4 x_{0} \Gamma_{0} \int \frac{d^{2} p_{\perp} d \xi}{\xi} f\left(\xi, p_{\perp}^{2}\right) d \tau,
$$

where $\Gamma_{0}=\frac{\alpha G_{F}^{2}}{32 \pi^{4}}\left|V_{t b} V_{t s}\right|^{2} c_{7}^{2}\left(m_{b}\right) \bar{m}_{b}(\mu)^{2} m_{b}^{3}$ is the contribution of the matrix element of $O_{7}$ to the $b \rightarrow s \gamma$ decay rate, and $x_{0}=m_{b} / M_{B}$. In HQET the mass $m_{b}$ is usually associated with the $b$ quark pole mass $m_{b, p o l e}$. In our phenomenological consideration we associate $m_{b}$ with the constituent mass of the $b$-quark, see below. The normalization of $f\left(\xi, p_{\perp}^{2}\right)$ reads $\pi \int_{0}^{1} d \xi \int_{0}^{\infty} d k_{\perp}^{2} f\left(\xi, k_{\perp}^{2}\right)=1$, where the factor $1 / \xi$ comes from the normalization of the $\bar{B} \rightarrow b \bar{d}$ vertex [1] $\overline{1}_{1}$. The phase space factor $d \tau$ is given by

$$
d \tau=\delta\left[\left(p_{b}-q\right)^{2}\right] E d E .
$$

We choose the $\mathrm{z}^{-}$-axis parallel to the 3 -vector $\mathbf{q}$, so that $q_{+}=2 E, q_{-}=0$, where $q_{ \pm}=q_{0} \pm q_{z}$, then $d \tau$ takes the form

$$
d \tau=\delta\left(m_{b}^{2}-\frac{p_{\perp}^{2}+m_{b}^{2}}{p_{b}^{+}}\right) E d E .
$$

In the first approximation we neglect $p_{\perp}^{2}$ in the argument of the $\delta$-function. Then, introducing the scaling variable $y=2 E / M_{B}$, the photon spectrum $d \Gamma / d y$ in $\bar{B} \rightarrow X_{s} \gamma$ takes the simple form

$$
\frac{1}{\Gamma_{0}} \cdot \frac{d \Gamma\left(\bar{B} \rightarrow X_{s} \gamma\right)}{d y}=R_{L F}(y),
$$


where

$$
R_{L F}(y)=\frac{1}{x_{0}} y \tilde{f}(y),
$$

and $\tilde{f}(\xi)=\pi \int_{0}^{\infty} d p_{\perp}^{2} f\left(\xi, p_{\perp}^{2}\right)$. Equivalently, one can write the spectrum in the standard QCD form $\left.11 / \Gamma_{0}\right) d \Gamma / d E=(2 / \Lambda) F(x)$. Therefore the specific choice adopted for $\tilde{f}(\xi)$ corresponds to a particular form of the QCD shape function $F(x)=\left(\Lambda / M_{B} x_{0}\right) y \tilde{f}(y)$, where $x=$ $\left(y-x_{0}\right) /\left(1-x_{0}\right)$.

Following Ref. [1] 3in we now account for the transverse motion of the $b$-quark in Eq. (2.4) to find a bit more complicated expression for $R_{L F}(y)[1]$

$$
R_{L F}(y)=2 m_{b} \pi \int_{y}^{1} f\left(\xi, p_{\perp}^{* 2}\right) d \xi
$$

where the integration limits follow from the condition $p_{\perp}^{* 2} \geq 0$, with $p_{\perp}^{* 2}=p_{\perp}^{* 2}(\xi, E)=m_{b}^{2}(\xi / y-$ $1)$. In this case the shape of the spectrum is obtained by direct integration of the distribution function. The difference between $R_{L F}(y)$ given by equations (2.6i) and (2.7) is very small numerically $\overline{1} \overline{1} \bar{j}$.

In the free quark approximation, $f\left(\xi, p_{\perp}^{2}\right)=$ $\delta\left(\xi-\xi_{0}\right) \delta\left(p_{\perp}^{2}\right)$, the total inclusive width $\Gamma(\bar{B} \rightarrow$ $\left.X_{s} \gamma\right)$ is the same as the radiative $b \rightarrow s \gamma$ width $\Gamma_{0}$. In this approximation $B \rightarrow X_{s} \gamma$ is a twobody decay (at leading order in $\alpha_{s}$ ) and the photon spectrum is monochromatic:

$$
\frac{d \Gamma_{0}}{d E}=\Gamma_{0} \delta\left(E-\frac{m_{b}}{2}\right) .
$$

The delta-function of equation (2.81) is transformed into a peak of a finite width due to the heavy quark motion. This effect is solely responsible for the filling in the windows between $m_{b} / 2$ and the kinematical boundary in the $B$ meson decay, $E_{\max }=M_{B} / 2 .^{2}$. The expressions in equations $(2.61),(2.7)$ exhibit a pronounced peak which is rather asymmetric. It is gratifying feature of the LF model, since it is in qualitative accord both with findings in QCD and experimental data. The perturbative corrections arising from gluon

\footnotetext{
${ }^{2}$ The true endpoint is actually located at $\left[M_{B}^{2}-\right.$ $\left.\left(m_{K}+m_{\pi}\right)^{2}\right] / 2 M_{B} \approx 2.60 \mathrm{GeV}$, i.e. slightly below $M_{B} / 2 \approx 2.64 \mathrm{GeV}$.
}

Bremsstrahlung and one-loop effects [20'] also lead to a nontrivial photon spectrum at the partonic level. Since our primary object here is to discuss non-perturbative effects due to the Fermi motion, we will implicitly ignore perturbative gluon emission throughout our analysis. In this case the parton matrix element squared is a constant and can be taken out of the integral in equation $(2 \cdot 2)$.

\section{Ansätzes for the LF Distribution Function}

We shall proceed by making an ansätz for the momentum space structure of the wave function. This is model dependent enterprise but has its close equivalent in studies of $\bar{B} \rightarrow X_{s} \gamma$ using the ACM model. In what follows, we will adopt both a phenomenological LF wave function and the LF functions corresponding to the various equal time (ET) quark model wave functions. As to the phenomenological ansätz, we use a model first written in Ref. [2]

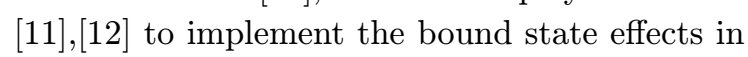
$B$-meson decays. It is written in the Lorentzinvariant form

$$
\psi(|\mathbf{p}|)=\mathcal{N} \exp \left(-\frac{\lambda}{2} v_{B} v_{s p}\right)=\mathcal{N} \exp \left(-\frac{\lambda}{2} \frac{\varepsilon_{p}}{m_{s p}}\right),
$$

where $v_{B}$ and $v_{s p}$ are the 4 -velocities of the $b$ quark and the quark-spectator, respectively, and $\varepsilon_{p}=\sqrt{|\mathbf{p}|^{2}+m_{s p}^{2}}$ is the energy of the spectator. We shall use the normalization condition $\int_{0}^{\infty} p^{2} d p \psi^{2}(|\mathbf{p}|) / 2 \varepsilon_{p}=1$, in which case one obtains $\mathcal{N}^{2}=2 \lambda /\left(m_{s p}^{2} K_{1}(\lambda)\right)$, where $K_{1}(\lambda)$ is the McDonald function. The function $\Phi\left(p^{2}\right)=\psi^{2}(|\mathbf{p}|) / 2 \varepsilon_{p}$ represents a momentum distribution of the spectator quark in the $B$ meson rest frame. We convert from ET to LF momenta by leaving the transverse momenta unchanged and letting

$$
p_{i z}=\frac{1}{2}\left(p_{i}^{+}-p_{i}^{-}\right)=\frac{1}{2}\left(p_{i}^{+}-\frac{p_{i \perp}^{2}+m_{i}^{2}}{p_{i}^{+}}\right)
$$

for both the $b$-quark $(i=b)$ and the quarkspectator $(i=s p)$. The longitudinal LF momentum fractions $\xi_{i}$ are defined as $\xi_{s p}=p_{s p}^{+} / P_{B}^{+}$, 
$\xi_{b}=p_{b}^{+} / P_{B}^{+}$, with $p_{b}^{+}+p_{s p}^{+}=P_{B}^{+}$. In the $B-$ meson rest frame $P_{B}^{+}=M_{B}$. Then for the distribution function $f\left(\xi, p_{\perp}^{2}\right)\left(\xi=1-\xi_{b}\right)$ one obtains

$$
\begin{array}{r}
f\left(\xi, p_{\perp}^{2}\right)=\frac{1}{8 \pi} \cdot \frac{\psi^{2}\left(\xi, p_{\perp}^{2}\right)}{1-\xi}=\frac{\mathcal{N}^{2}}{8 \pi(1-\xi)} \\
\times \exp \left[-\frac{\lambda}{2}\left(\frac{1-\xi}{\xi_{0}}+\frac{\xi_{0}}{1-\xi}\left(1+\frac{p_{\perp}^{2}}{m_{s p}^{2}}\right)\right)\right],
\end{array}
$$

where $\xi_{0}=m_{s p} / M_{B}$. The function (3.3is is sharply peaked at $p_{\perp}^{2}=0, \xi=\xi_{0}$. In what follows, we shall refer to the LF wave function of equation (3.3) as the case A.

A priory, there is no connection between the ET momentum distribution $\Phi\left(p^{2}\right)$ of a constituent quark model and LF wave function $\psi\left(x, p_{\perp}^{2}\right)$. However, the mapping between the variables described above turns a normalized solution of the ET equation of motion into a normalized solution of the different looking LF equation [22-1]. Because the ET function depends on the relative momentum it is more convenient to use the quark-antiquark rest frame instead of the $B$-meson rest frame. Recall that in the LF formalism these two frames are different. As a result one obtains the $\mathrm{LF}$ wave function as $\psi\left(\xi, p_{\perp}^{2}\right)=\left(\partial p_{z} / \partial \xi\right) \Phi\left(p_{\perp}^{2}+\right.$ $\left.p_{z}^{2}\left(\xi, p_{\perp}^{2}\right)\right)$. Explicit form of this function is given e.g. by equation (10) of Ref. [1 127 . It is wave functions made kinematically relativistic in this fashion, that were used in a recent calculation of the $B_{c}$ lifetime $[\overline{2} \overline{3} \rrbracket$. We calculate the photon energy spectra using the three representative LF wave functions corresponding to the non-relativistic ISGW2 [24] constituent quark models ${ }^{3}$. The main difference between the ET wave functions of these models relies in the behaviour at high value of the internal momentum, for further discussion see [2 $\left.23^{\prime}\right]$. We believe that the spread of results obtained for these distribution functions is a fair representation of model dependence resulting from the inclusion of Fermi motion.

Having specified the non-perturbative aspects of our calculations, we proceed to present numer-

\footnotetext{
${ }^{3}$ The ET ISGW2 function corresponds to the Gaussian distribution $\Phi\left(p^{2}\right)$ conventially employed in the ACM model with $p_{F}=0.43 \mathrm{GeV}$. For AL1 and DSR models we use simple analytical parametrizations of the ET wave functions [27].
}

ical results for the photon spectrum in the decay $B \rightarrow X_{s} \gamma$. In case A we take $m_{b}=4.8 \mathrm{GeV}$, $m_{s p}=0.3 \mathrm{GeV}$ and $\lambda=2$ as reference values. These values are motivated by a study of the $b \rightarrow c$ decays $[127]$. For the models $\mathrm{B}$ to $\mathrm{D}$ we use the constituent quark masses listed in table 1 .

Table 1 . The values of the constituent quark mass $m_{b}$ and $m_{s p}$ (in units of $\mathrm{GeV}$ ) for the models $\mathrm{A}$ to $\mathrm{D}$. The values of $\tilde{m}_{b}$ as defined by the condition $\langle x\rangle=0$ are also indicated.

\begin{tabular}{|c|c|c|c|c|}
\hline \hline Model & $\mathrm{A}$ & $\mathrm{B}$ & $\mathrm{C}$ & $\mathrm{D}$ \\
\hline$m_{b}$ & 4.80 & 5.20 & 5.227 & 5.074 \\
\hline$\tilde{m}_{b}$ & 4.73 & 4.68 & 4.73 & 4.60 \\
\hline$m_{s p}$ & 0.30 & 0.33 & 0.315 & 0.221 \\
\hline \hline
\end{tabular}

The choice of $m_{b}$ in our approach deserves some comments. The numerical calculations using the constituent $b$-quark masses show large deviations of the $\Gamma\left(\bar{B} \rightarrow X_{s} \gamma\right)$ from the free decay rate $\Gamma_{0} \propto m_{b}^{3}(\approx 10 \%$ for the cases $\mathrm{B}$ to $\mathrm{D}$, see table 2$)$. This signals the appearence of the linear $1 / m_{b}$ corrections to the free quark limit. The reason is that the constituent quark models usually employ the $b$-quark masses that are $300-400 \mathrm{MeV}$ higher than the pole $b$-quark mass. This fact seems to be a subtlety in applying the constituent quark model approach to calculate the non-perturbative corrections to the $\bar{B} \rightarrow X_{s} \gamma$ inclusive rate. To overcome the uncertainties induced by the constituent mass of the $b$ quark we use a simple phenomenological receipe that considerably improves the situation. Notice that, as in the ACM model [i] $\left.\overline{1}_{1}^{\prime}\right], 1 / m_{b}$ corrections can be absorbed into the definition of the $b$-quark mass. We introduce $\tilde{m}_{b}=m_{b}+\delta m_{b}$ by imposing the condition $\bar{y}\left(\tilde{m}_{b}\right)=x_{0}$, where $\bar{y}=\int_{0}^{1} y R_{L F}(y) d y$. This condition coincides with that used in HQET to define the pole mass of the $b$-quark. As a result, the correction to first order in $1 / m_{b}$ will be eliminated from the total width in agreement with the general statement of HQET. To illustrate our arguments, consider the analytically tractable case of the photon spectrum of equation $\left({ }_{2}^{2} . \overline{6}\right)$ with the distribution func- 
tion given by equation (3.3i). In this case $\tilde{f}(\xi)=$ $2 \lambda /\left(\xi_{0} K_{1}(\lambda)\right) \exp \left(-\lambda / 2\left[\xi_{0} /(1-\xi)+(1-\xi) / \xi_{0}\right]\right)$, and simple analytical expressions for the integrated spectrum $R_{L F}=\int_{0}^{1} R_{L F}(y) d y$ and $\bar{y}$ are avaiable,

$R_{L F}=\frac{1}{x_{0}}\left(1-\xi_{0} \kappa_{2}\right), \quad \bar{y}=\frac{1}{x_{0}}\left(1-2 \xi_{0} \kappa_{2}+\xi_{0}^{2} \kappa_{3}\right)$,

where $\kappa_{n}=K_{n}(\lambda) / K_{1}(\lambda)$. The $1 / M_{B}$ correction to $R_{L F}$ can be absorbed into the definition of $\tilde{m}_{b}$. Indeed, neglecting $\xi_{0}^{2}$ and letting $\tilde{m}_{b} \approx(1-$ $\left.\xi_{0} \kappa_{2}\right) M_{B}$, one obtains $R_{L F}=1+O\left(1 / M_{B}^{2}\right)$. The $B$-meson mass can be eliminated in favour of the b-quark mass, so we have the desired result, $R_{L F}=1+O\left(1 / m_{b}^{2}\right)$.

We have calculated numerically the values of $\tilde{m}_{b}$ in different models using equation (2.7i) for $R_{L F}(y)$. Although $\tilde{m}_{b}$ depend on the assumed shape of distribution, this dependence is marginal: the uncertainty on $\tilde{m}_{b}$ is between 4.6 and $4.7 \mathrm{GeV}$, depending on the choice of $f\left(\xi, p_{\perp}^{2}\right)$, see table 1 . These values are consistent whith the $b$ quark pole mass $m_{b, p o l e}=4.8 \pm 0.15 \mathrm{GeV}$ [2 $\left.{ }^{2} \overline{2}^{\prime}\right]$. If we repeat the same exercise by applying equation $(2.6)$ we find practically identical values of $\tilde{m}_{b}$.

\section{The Photon Energy Spectra}

We first study the photon energy spectra. Here, and henceforth, we present the results obtained using equation $\left(2 . \overline{7}_{1}\right)$. Our results for the photon spectra and moments are reported in figure 1 and table 2. The different curves in figure 1 correspond to the models $\mathrm{A}$ to $\mathrm{D}$. The influence of the various choices of the distribution function and the $b$-quark mass can be read off from table 2, where we show both $R_{L F}\left(m_{b}\right)$, $R_{L F}\left(\tilde{m}_{b}\right)$ and analogous quantities $R_{A C M}$ calculated in the ACM model, see below. In table 2 we also show the average photon energy $\bar{y}$ normalized to $M_{B} / 2$, and the moments $\overline{y^{2}}$ and $\overline{y^{3}}$. The substitution $m_{b} \rightarrow \tilde{m}_{b}$ modifies the predictions for the total rate and the moments $\bar{y}, \overline{y^{2}}$ by about $8-9 \%$ for the models $\mathrm{B}-\mathrm{D}$, while it affects the corresponding quantities by less than $1.5 \%$

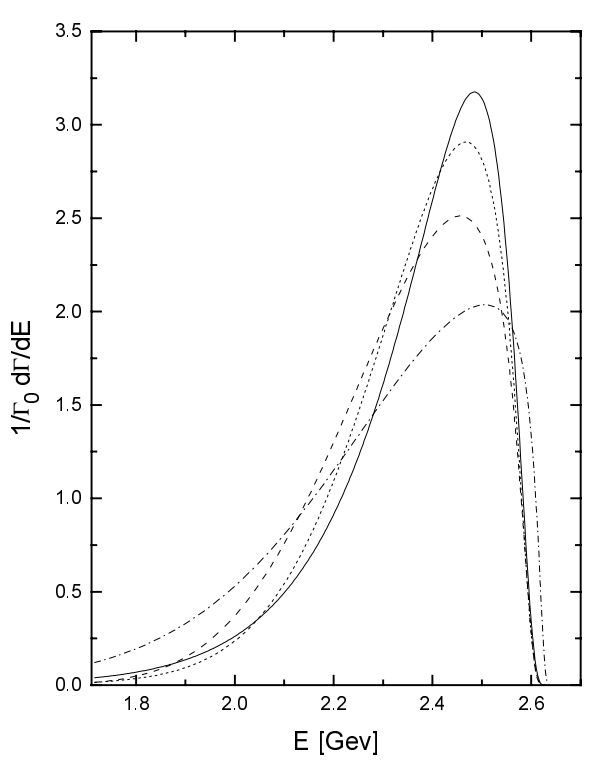

Figure 1: Theoretical predictions for the photon energy spectra using the LF quark models described in the text. The solid, dashed, dot-dashed, and dotted curves correspond to the models A to D, respectively. The spectra are calculated using equation (2.7.)

for the model A. Our final results for the integrated photon spectra $R_{L F}\left(\bar{m}_{b}\right)$ agree well with the corresponding OPE prediction [in $R_{\mathrm{OPE}}=$ $1+\left(\lambda_{1}-9 \lambda_{2}\right) / 2 m_{b}^{2}$, where $\lambda_{1}$ and $\lambda_{2}$ parameterize the matrix elements of the kinetic and chromomagnetic operators, respectively. For $\lambda_{1}=$ $-0.3 \pm 0.2 \mathrm{GeV}^{2}$ and $\lambda_{2}=0.12 \pm 0.02 \mathrm{GeV}^{2}$ $R_{\mathrm{OPE}}$ affects the free quark result by a few per cent, $R_{\mathrm{OPE}}=0.975 \pm 0.005$.

The dependence of the energy moments $\bar{y}^{n}$ on $m_{b}$ is rather weak, in contrast to that of the QCD moments of the shape function $\left\langle x^{n}\right\rangle=$ $\int_{-2 M_{B} / \bar{\Lambda}}^{1} x^{n} F(x) d x$, which are very sensitive to the difference between $M_{B}$ and $m_{b}$. In particular, changing the $b$-quark mass from $m_{b}$ to $\tilde{m}_{b}$ modifies $\left\langle x^{2}\right\rangle$ and $\left\langle x^{3}\right\rangle$ dramatically. Note that the resulting values of $\left\langle x^{2}\right\rangle$ and $\left\langle x^{3}\right\rangle$ still have a sizable model dependence. Our predic- 
tions for $\left\langle x^{2}\right\rangle$ are somewhat small, although in agreement (for the model D) with the result of Ref. [i] and compatible with the results obtained from the QCD sum rules, $\left\langle x^{2}>\approx 0.5\right.$. This means that the LF ansatz can be made consistent with the QCD description provided the spectator quark is relativistic. This conclusion agrees with the similar conclusion obtained for the ACM model in Ref. [1] $\overline{1}$ in.

Table 2. The total integrated rates $R_{L F}$, $R_{A C M}$ calculated in the LF and ACM models, respectively, the moments of the photon energy spectrum $\bar{y}^{n}, n=1,2,3$, and $\left\langle x^{2}\right\rangle,\left\langle x^{3}\right\rangle$ for the cases A to D. The scaling variables $x$ and $y$ are defined in the text. The results have been obtained using equation (2.7i).

\begin{tabular}{|c|c|c|c|c|}
\hline \hline Model & $\mathrm{A}$ & $\mathrm{B}$ & $\mathrm{C}$ & $\mathrm{D}$ \\
\hline$R_{L F}\left(m_{b}\right)$ & 0.973 & 0.900 & 0.905 & 0.899 \\
\hline$R_{L F}\left(\tilde{m}_{b}\right)$ & 0.987 & 0.986 & 0.989 & 0.974 \\
\hline \hline$R_{\mathrm{ACM}}\left(\tilde{m}_{b}\right)$ & 1.008 & 1.005 & 1.008 & 1.003 \\
\hline \hline $\bar{y}\left(m_{b}\right)$ & 0.873 & 0.806 & 0.818 & 0.795 \\
\hline $\bar{y}\left(\tilde{m}_{b}\right)$ & 0.885 & 0.873 & 0.885 & 0.851 \\
\hline $\bar{y}^{2}\left(m_{b}\right)$ & 0.787 & 0.726 & 0.742 & 0.709 \\
\hline $\bar{y}^{2}\left(\tilde{m}_{b}^{2}\right)$ & 0.798 & 0.778 & 0.796 & 0.751 \\
\hline $\bar{y}^{3}\left(m_{b}\right)$ & 0.712 & 0.655 & 0.676 & 0.636 \\
\hline $\bar{y}^{3}\left(\tilde{m}_{b}\right)$ & 0.722 & 0.695 & 0.718 & 0.669 \\
\hline \hline$<x^{2}>$ & 0.378 & 0.295 & 0.302 & 0.456 \\
\hline$<-x^{3}>$ & 0.348 & 0.130 & 0.176 & 0.348 \\
\hline \hline
\end{tabular}

In order to compare our results with those of the ACM model we have calculated the inclusive $\bar{B} \rightarrow X_{s} \gamma$ photon spectra in a simplified ACM model [1] bution (2.8) for the free $b$-quark. We have used the momentum distributions $\Phi\left(p^{2}\right)$ of the spectator quark for the models A to D. In all cases we have found that the spectra calculated in ACM and LF parton models are almost identical. This is not surprising because we have checked numerically that the quark masses $\tilde{m}_{b}$ defined using the LF models practically coincide with the values of the floating $b$ quark mass $m_{b}^{f}=M_{B}-\sqrt{m_{s p}^{2}+p^{2}}$ averaged over the distribution $\Phi\left(p^{2}\right)$. The integrated energy spectra $R_{A C M}$ for the models A to $\mathrm{D}$ are reported in table 2 , they coincide with

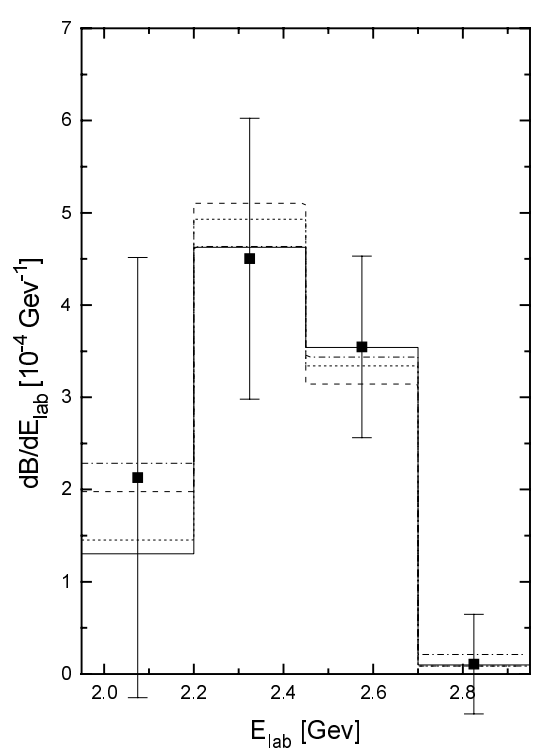

Figure 2: Theoretical predictions for the photon energy spectrum in the laboratory frame for the different distribution functions $f\left(\xi, p_{\perp}^{2}\right)$. The notations are the same as in figure 1 . The data points show the CLEO results. The curves show the results of the best fit reported in table 3 .

$R_{L F}$ within a per cent accuracy.

Although we do not consider here perturbative corrections, it is instructive to compare theoretical predictions for the Doppler shifted spectrum $d B / d E_{l a b}$ in the laboratory frame with the CLEO data. To perform a fit to the data, we rebin the boosted photon spectra in the same energy intervals as used by CLEO and, for each choice of the distribution function, adjust the overall normalization to give the best fit to the data. The results are reported in table 3 , and the best fits are displayed in figure 2. All fits have $\chi^{2} / n_{\text {dof }} \ll 1$, indicating the present accuracy of experiment. Averaging over the models we obtain

$$
B\left(\bar{B} \rightarrow X_{s} \gamma\right)=\left(2.5 \pm 0.5_{\text {exp }} \pm 0.3_{\text {model }}\right) \times 10^{-4},
$$

where the last error comes from the model dependence. This result is consistent with the update CLEO measurement of the inclusive $b \rightarrow s \gamma$ rate 
$[2 \overline{2} 9 \overline{9}] B\left(\bar{B} \rightarrow X_{s} \gamma\right)=(3.15 \pm 0.35 \pm 0.41) \times 10^{-4}$.

Finally, we note that the invariant mass $M_{H}$ of the hadronic final state is related with the scaling variable $y$ by $M_{H}^{2}=M_{B}^{2}(1-y)$. Therefore the theoretical results for the photon spectrum can be translated into predictions for the hadronic mass spectrum. In figure 3 we show the invariant mass distribution of the hadrons recoiling against the photon for the models A-D. Our predictions for hadronic mass spectra must be understood in the sence of quark-hadron duality. The true hadronic mass spectrum for low $M_{H}^{2} \propto M_{K^{*}}^{2}$ may have resonance structure that looks rather different from our predictions. A realistic model for the hadronic mass spectrum consists of a single peak located at the mass $K^{*}(892)$ followed by a continuum (above a threshold value $M_{t h}$ ) which is given by the inclusive spectrum and is dual to a large number of overlapping resonances. In table 3 we show the ratios $R_{K^{*}(892)}=B(\bar{B} \rightarrow$ $\left.K^{*} \gamma\right) / B\left(\bar{B} \rightarrow X_{s} \gamma\right)$ obtained by the integration of the inclusive spectrum in the range $M_{H} \leq$ $M_{t h}$. The result crucially depends on the choice of $M_{t h}$; we use the value of $M_{t h}=1.15 \mathrm{GeV}$ adopted in Ref. [i]n. Averaging over the different model predictions we obtain $R_{K^{*}(892)}=$ $0.157_{-0.44}^{+0.24}$. This result agrees both with other theoretical predictions and with the CLEO measurement $R_{K^{*}(892)}^{\exp }=0.17 \pm 0.08$.

Table 3. The branching ratios $B\left(\bar{B} \rightarrow X_{s} \gamma\right)$ obtained from the fit to the CLEO data and the partial fractions $R_{K^{*}(892)}$.

\begin{tabular}{|c|c|c|c|c|}
\hline \hline Model & $\mathrm{A}$ & $\mathrm{B}$ & $\mathrm{C}$ & $\mathrm{D}$ \\
\hline$\Gamma_{0} / \Gamma_{B} \cdot 10^{4}$ & 2.54 & 2.59 & 2.50 & 2.91 \\
\hline$R_{K^{*}(892)}$ & 0.1681 & 0.1312 & 0.1486 & 0.1807 \\
\hline \hline
\end{tabular}

\section{Conclusions}

We have derived a new parton formula, which establishes a simple connection between the electroweak decay rate $\Gamma\left(\bar{B} \rightarrow X_{s} \gamma\right)$ and the rate of a free $b$-quark decay. Our main result is equations

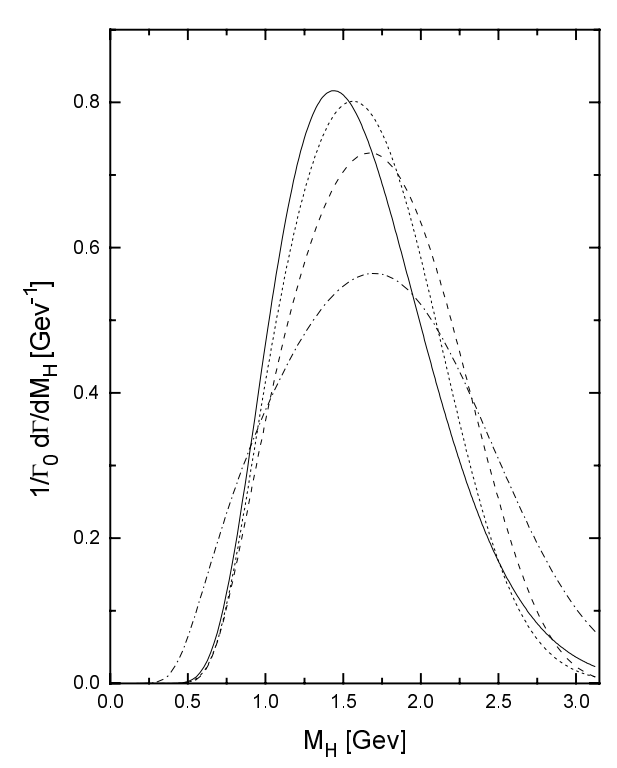

Figure 3: Theoretical predictions for the invariant hadronic mass spectrum for different distribution functions $f\left(\xi, p_{\perp}^{2}\right)$. The notations are the same as in figure 2 .

$(2.6),(2.7)$. Using various $b-$ quark distribution functions we have calculated the photon energy spectra and the corrections to the free decay rate. We have shown that the decay width has no linear to $1 / m_{b}$ corrections only if expressed not in terms of the constituent quark mass but in terms of a mass $\tilde{m}_{b}$ which is defined in the way similar to that used in HQET to define $m_{b, \text { pole. }}$. In this way one avoids an otherwise large (and model dependent) correction of order $1 / m_{b}$ but at expence of introducing the shift in the constituent quark mass which largely compensates the bound state effects. A summary of our results presented in tables 2,3 shows a fair good agreement both with the QCD results and avaiable experimental data. We have also found that the photon energy spectra calculated in our LF parton-like approach agree well with the ones obtained in the ACM model, provided the same ET distribution function $\Phi\left(p^{2}\right)$ is used as input in both cases. It would be interesting to check whether the effective values of the $b$-quark mass $\tilde{m}_{b}$ can appear to 
be approximately the same for different channels $(b \rightarrow c$ vs. $b \rightarrow u$ or $b \rightarrow s$ ) and for different beauty hadrons. This work is in progress, and the results will be reported elsewhere. I

\section{Acknowledgments}

This talk is based on work done together with Y., - Y.Keum,-P_Yu_Kulikov and _H.S.Song- - I szould '- tike t $\Theta$-thank -the -organizers- $-f-$ the- symap for their invitation and hospitality. This work was done in part under the INTAS grant Ref. No 96-155.

\section{References}

[1] S.Glenn et al, CLEO Collaboration, CLEO CONF $98-17$

_ [2]_A.Falk, M.Luke, M.Savage, Phys. Rev. D 49: ' - - (1994) 3367;

[3] A.Kapustin, Z.Ligeti, 'Phys. Lett. B $\mathbf{3 5 5}(1995)$, - 318

[4] J.Chay, H.Georgy, B.Grinstein, 'Phys. Lett.'

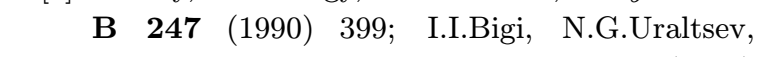

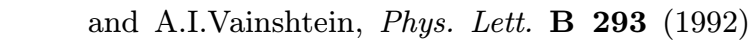
$===430 ;$ I.I.Bigi, M.Shifman, N.G.Uraltsev, and A.I.Vainshtein, 'Phys. Rev. Lett. 71 (1993) 496", A.V.Manokhar and M.B.Wise, $P$ Phys. Rev. D 49 $(1994) 1310$

[5] M.Neubert, 'Phys. Rev. D $\mathbf{4 9}$ (1994) 3392, Phys.' Rev. D $\mathbf{4 9}$ (1994) 4623; T.Mannel and M.Neubert, ,Phys. Rev. D $\mathbf{5 0}(1994)$ 2037; I - - - I.I.Bigi, _ - M. I--_-_.I.Vainstein. 'Int. (1994) 2467; R.D.Dikeman, M.A.Shifman, and

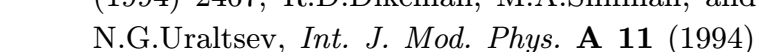

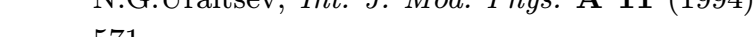
(1) $=-5 \overline{1}$

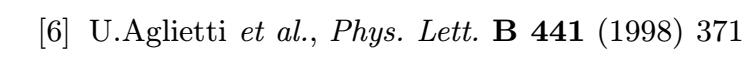

[7] A.L.Kagan, M.Neubert, Euro. Phys. J. C7 (1999) 5

_[8] A.Ali, E.Pietarinen, 'ucl. Phys. B 154 1979$)_{1}^{\prime}$ - - 512

[9] G.Altarelli, N.Cabbibo, G.Corbo, L.Maiani, and G.Martinelli, 'Nucl. Phys. B 202 (1982) 512, [10]_C.H.Jin, M.F.Palmer, and E.A.Paschos, iPhys.' - _ _ett. B_329(1994)_364:
[11] V.L.Morgunov, K.A.Ter-Martirosyan, Phys. Atom. Nucl. 59 (1996) 1221

[12] I.L.Grach, I.M.Narodetskii, S.Simula, and K.A.Ter-Martirosyan, 'Nucl. Phys. B $\mathbf{5}$ (1997) 2227

[13] S.Kotkovsky, I.M.Narodetskii, S.Simula, and K.A.Ter-Martirosyan, hep-ph/9712543, to appear in Phys. Rev. D

[14] S.Kotkovsky, I.M.Narodetskii, K.A.TerMartirosyan, and S.Simula, 'Nucl. Phys. $\overline{\mathbf{7}} \mathbf{-} \overline{\mathrm{B}}$ (Proc. Suppl.) (1999) 100'

[15] A.Vainstein, V.Zakharov and M.Shifman, Pis'ma v ZHETF 22 (1975) 123 [JETP Lett.

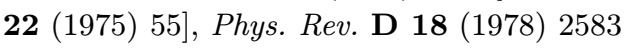

[16] T.Inami and C.S.Lim, Prog. Theor. Phys. 65 (1981) 297; B.A.Campbell and P.J.O'Donnell, Phys. Rev. D_25 (1982)_1989

[17] N.B.Demchuck, I.L.Grach, I.M.Narodetskii, and S.Simula, Phys. At. Nucl. 59 (1996) 2152

[18] I.Bigi, M.Shifman, N.Uraltsev, A.Vainstein, Phys. Lett. B 328 (1994) 431'

[19] Y.-Y.Keum, P.Yu.Kulikov, I.M.Narodetskii, H.S.Song, hep-ph/9906416, to appear in Phys. Lett. B

[20] A.Ali and C.Creub, 'Z. Physik C $\mathbf{4 9}$ (1991) 431', ibid. 60 (1993) 433, 'Phys. Lett. B 229_(1991)' $182_{\mathrm{v}}^{1}$ ibid. 287 (1992) 191, ibid. $\mathbf{3 6 1}$ (1995) 146, N.Pott, 'Phys. Rev. D $\mathbf{5 4}(\overline{1995)} 93 \overline{1}$

[21] A.Dubin and A.Kaidalov, Yad. Fiz. 56 (1993) 164 [Phys. At. Nucl. 56 (1993) 237]

[22] F.Coester, Prog. Part. Nucl. Phys. 29 (1992) 1

[23] A.Anisimov, I.M.Narodetskii, C.Semay, and B.Silvestre-Brac, 'Phys. Lett. B 452 (1999) $129_{1}^{\prime}$

[24] D.Scora and N.Isgur, Phys. Rev. D $\mathbf{5 2}(1995)$ 2783

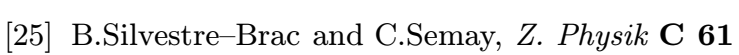
$(199 \overline{4}) \overline{2} \overline{1}$ 1, Few-Body Systems 20 (1996) 1

[26] B.Silvestre-Brac and C.Semay, '№cl. Phys. A' $\mathbf{2 2 1}(1997) 455$

[27] C.Semay and B.Silvestre-Brac, private communication

[28] M.Neubert, Phys. Rep. 245 (1994) 259

[29] T.Skwarnicki, in Proceedings of ICHEP'98, Vancouver, B.C., Canada, 1998, Eds. A.Astbury, D.Axen and J.Robinson, World Scientific, p. 1057 